\title{
Sleep-Dependent $\theta$ Oscillations in the Human Hippocampus and Neocortex
}

\author{
Jose L. Cantero, ${ }^{1}$ Mercedes Atienza, ${ }^{1}$ Robert Stickgold, ${ }^{1}$ Michael J. Kahana, ${ }^{2}$ Joseph R. Madsen, ${ }^{3}$ and Bernat Kocsis ${ }^{1}$ \\ ${ }^{1}$ Laboratory of Neurophysiology, Department of Psychiatry, Massachusetts Mental Health Center, Harvard Medical School, Boston, Massachusetts 02115, \\ ${ }^{2}$ Volen Center for Complex Systems, Brandeis University, Waltham, Massachusetts 02454, and 'Department of Neurosurgery, Children's Hospital, and \\ Department of Surgery, Harvard Medical School, Boston, Massachusetts 02115
}

\begin{abstract}
Hippocampal $\theta$ waves recorded during rapid eye movement (REM) sleep are thought to play a critical role in memory consolidation in lower mammals, but previous attempts to detect similar $\theta$ oscillations in the human hippocampus have been unsuccessful. Using subdural and depth recordings from epileptic patients, we now report the first evidence of state-dependent hippocampal $\theta$ waves (4-7 $\mathrm{Hz}$ ) in humans. Unlike the continuous $\theta$ in rodents, however, these oscillations were consistently observed during REM sleep in short $(\sim 1$ sec) bursts and during transitions to wake in longer epochs. $\theta$ waves were also observed in the basal temporal lobe and frontal cortex during transitions from sleep to wake and in quiet wakefulness but not in REM, and they were not coherent with hippocampal $\theta$ oscillations. The absence of functional coupling between neocortex and hippocampus during $\theta$ periods indicates that multiple $\theta$ generators exist in the human brain, and that they are dynamically regulated by brain state. $\gamma$ oscillations were also present during REM $\theta$ bursts, but the fluctuations in $\gamma$ power were not associated with $\theta$ phase, pointing out another significant difference between rodent and human $\theta$ properties. Together, these findings suggest that the generation mechanisms of $\theta$ oscillations in humans might have evolved from tonic to phasic in hippocampus during REM sleep and extended from hippocampus to cortex, where they appear in certain wakefulness-related states.
\end{abstract}

Key words: $\theta$ oscillations; hippocampus; cerebral cortex; sleep; intracranial recordings; humans

\section{Introduction}

$\theta$ oscillations are one of the most prominent electrophysiological features of the mammalian hippocampus (Whishaw and Vanderwolf, 1973; Vertes and Kocsis, 1997; Buzsaki, 2002;). They have been hypothesized to be involved in memory encoding and retrieval (O'Keefe and Recce, 1993; Skaggs et al., 1996), locomotor activities in rodents (Vanderwolf, 1969), sensorimotor integration (Bland and Oddie, 2001), learning rate (Berry and Thompson, 1978; Seager et al., 2002), and long-term potentiation (Larson and Lynch, 1986; Pavlides et al., 1988; Huerta and Lisman, 1996; Holscher et al., 1997). In lower mammals, hippocampal $\theta$ oscillations have demonstrated to be a reliable correlate of certain behavioral states, emerging as a fundamental electrophysiological feature of rapid eye movement (REM) sleep and specific behaviors during wakefulness (Winson, 1972).

Previous studies have reported human hippocampal $\theta$ modulations related to behavior during wakefulness (Halgren et al., 1978; Arnolds et al., 1980; Meador et al., 1991). Two of these studies, performed both with a single case, showed opposite re-

\footnotetext{
Received July 30, 2003; revised Sept. 15, 2003; accepted Sept. 16, 2003.

This work was supported by a postdoctoral fellowship from the Spanish Ministry of Science and Technology (J.L.C.), a NATO Postdoctoral Fellowship (M.A.), and National Institutes of Health Grants MH-61975 to M.K., MH48832 and DA11744 to R.S., and MH-062525 to B.K. We thank D. Seelig and M. Tully for help with determining Talairach coordinates and CT images, respectively.

Correspondence should be addressed to Dr. Jose L. Cantero, University Pablo de Olavide, Ctra. de Utrera, Km. 1, 41013-Seville, Spain. E-mail: jlcanlor@dex.upo.es.

Copyright $\odot 2003$ Society for Neuroscience $\quad$ 0270-6474/03/2310897-07\$15.00/0
}

sults when voluntary motor behaviors were associated with hippocampal oscillations. Thus, Arnolds et al. (1980) found a higher spectral power within the $\theta$ band linked to certain motor behaviors, whereas Halgren et al. (1978) observed hippocampal $\theta$ suppression associated with the movement density. Hippocampal $\theta$ power was also found to be significantly higher during conditions of resting eyes-closed and learning of word-paired lists, as compared with resting eyes-open and visuospatial learning conditions (Meador et al., 1991). Furthermore, task-dependent $\theta$ waves have recently been described in human neocortex and were implicated in multiple mnemonic functions, including spatial navigation (Kahana et al., 1999; Caplan et al., 2003) and working memory (Raghavachari et al., 2001). Interestingly, these neocortical $\theta$ waves are usually short-lasting $(\sim 1-2 \mathrm{sec})$ and time-locked to the cognitive task (Rizzuto et al., 2003). In contrast, $\theta$ rhythms in lower mammals appear tonically in the hippocampus during periods of waking exploratory movements (Vanderwolf, 1969) as well as during REM sleep (Winson, 1972) and arousals from sleep (Green and Arduini, 1954).

Previous research using invasive EEG recordings from epileptic patients have failed to provide evidence of $4-7 \mathrm{~Hz} \theta$ activity in the human brain during sleep, although rhythmic activity from the $\theta$ range has been found in several studies (Freemon and Walter, 1970; Bodizs et al., 2001; Uchida et al., 2001). For instance, Bodizs et al. (2001) reported a significant increase in relative power within the $1.5-3 \mathrm{~Hz}$ band in parahippocampal gyrus during REM as compared with slow-wave sleep (SWS) and wake- 
Table 1. Summary of demographic and clinical information for each patient

\begin{tabular}{|c|c|c|c|c|c|c|c|}
\hline Patient & Gender & Age (years) & Seizure type & MRI findings & Implant type & Medication & Surgical procedure \\
\hline 1 & M & 15 & Left temporal lobe focus & None & Subdural plus depth & $\begin{array}{l}\text { Antibiotics, anticonvulsives, } \\
\text { analgesics }\end{array}$ & $\begin{array}{l}\text { Left lateral temporal lobe } \\
\text { and left mesial tem- } \\
\text { poral lobe resections }\end{array}$ \\
\hline 2 & M & 14 & $\begin{array}{l}\text { Right frontal and temporal } \\
\text { lobe focus }\end{array}$ & Chiari-I malformation & Subdural & Analgesics, anticonvulsives & $\begin{array}{l}\text { Anterior right temporal } \\
\text { and right frontal } \\
\text { resecctions }\end{array}$ \\
\hline 3 & M & 19 & Left temporal lobe focus & $\begin{array}{l}\text { Right choroidal cyst, cerebellar } \\
\text { tonsils below foramen } \\
\text { magnum }\end{array}$ & Subdural & Analgesics, anticonvulsives & $\begin{array}{l}\text { Left inferior temporal } \\
\text { lobe resection }\end{array}$ \\
\hline 4 & M & 15 & $\begin{array}{l}\text { Left mesial temporal lobe } \\
\text { focus }\end{array}$ & None & Depth & $\begin{array}{l}\text { Analgesics, anticonvulsives, } \\
\text { antibiotics }\end{array}$ & $\begin{array}{l}\text { Small resections of left } \\
\text { lateral temporal lobe, } \\
\text { hippocampus, and } \\
\text { amygdala }\end{array}$ \\
\hline 5 & M & 19 & Left occipital focus & Left temporoparietal lesions & Subdural plus depth & Analgesics, anticonvulsives & $\begin{array}{l}\text { Left occipital resection } \\
\text { and multiple subpial } \\
\text { transectiopns through } \\
\text { parieto-occipital } \\
\text { regions }\end{array}$ \\
\hline 6 & M & 10 & $\begin{array}{l}\text { Right parietotemporal } \\
\text { focus }\end{array}$ & None & Subdural & Analgesics, anticonvulsives & $\begin{array}{l}\text { Left temporal lobectomy } \\
\text { and left mesial hip- } \\
\text { pocampectomy }\end{array}$ \\
\hline 7 & M & 19 & Left parieto-occipital focus & $\begin{array}{l}\text { Dysplasia in the left posterior } \\
\text { frontal lobe }\end{array}$ & Subdural & Analgesics, anticonvulsives & $\begin{array}{l}\text { Left parieto-occipital } \\
\text { resection }\end{array}$ \\
\hline 8 & M & 11 & Left frontal focus & None & Subdural & Analgesics, anticonvulsives & $\begin{array}{l}\text { Left frontotemporal and } \\
\text { anterior frontal resec- } \\
\text { tions }\end{array}$ \\
\hline 9 & M & 11 & Left temporal lobe focus & None & Subdural & Analgesics, anticonvulsives & $\begin{array}{l}\text { Left anterior temporal } \\
\text { resection }\end{array}$ \\
\hline
\end{tabular}

fulness. Other authors have found faster activities $(10-20 \mathrm{~Hz})$ in the medial temporal lobe during both wakefulness and REM sleep but not in SWS (Uchida et al., 2001). Although the behavioral aspects of these findings are similar to the sleep-wake correlates of $\theta$ rhythm in animals, the difference in frequency range makes it premature to consider human oscillatory activities within the $\delta$ (Bodizs et al., 2001) and $\alpha-\beta$ (Uchida et al., 2001) bands equivalent to the animal hippocampal $\theta$ rhythm, or even to the state-dependent $\theta(4-7 \mathrm{~Hz})$ found in human EEG studies during waking and REM sleep.

To determine whether spontaneous $\theta$ oscillations appear in the human brain with the same state-dependent features as in rodents, we recorded electric field potentials across wake-sleep states from multiple subdural electrodes placed on cortical surfaces and depth electrodes implanted into the hippocampus. We found state-dependent $\theta$ oscillations $(4-7 \mathrm{~Hz})$ in the unfiltered EEG traces from hippocampus and different regions of neocortex. No functional coupling was seen between these two structures in any brain state, strongly suggesting that human hippocampus and neocortex have evolved to independent and state-dependent generators of $\theta$ waves.

\section{Materials and Methods}

Nine patients with drug-resistant epilepsy participated in this study (Table 1). All patients underwent invasive monitoring to localize the specific brain regions containing the epilepsy source. Intracranial electrodes were made from platinum discs ( $3 \mathrm{~mm}$ diameter) and placed on the cortical surface at $1 \mathrm{~cm}$ intervals. Depth electrodes were placed with a spacing of $8 \mathrm{~mm}$. Electrode impedance was typically $60-70 \Omega$ for both subdural and depth probes. Six patients had electrode arrays implanted unilaterally in various regions of the neocortex, two patients had bilateral depth electrodes placed within the hippocampus in combination with neocortical electrodes, and one patient had only bilateral depth electrodes within the hippocampus (Table 1). The research protocol was approved by the Committee on Clinical Investigation at Children's Hospital (Boston, $\mathrm{MA}$ ), and informed consent was obtained from the patients and their parents.

The location of the electrodes was determined using preoperative magnetic resonance imaging and was coregistered with postoperativecomputed tomograms by an indirect stereotactic technique (Talairach and Tournoux, 1988). Figure $1 A$ shows bilateral placement of depth electrodes within the hippocampal formation in one patient. Electrodes placed on the epileptic focus, overlying regions of radiographically evident structural damage, or exhibited epileptiform EEG (i.e., spikes or sharp waves, as determined by the clinical team) were excluded. When epochs containing EEG artifacts and epileptic activity were identified in a specific channel or set of channels, simultaneous EEG segments in the rest of the channels were also eliminated from additional analysis. To avoid maximal intromissions of oscillatory epileptic activity or artifact contamination, both $1 \mathrm{~min}$ before and $1 \mathrm{~min}$ after each EEG segment with artifacts and spikes was removed from the entire set of channels.

Intracranial and polysomnographic recordings were performed in the patient room from 10:00-11:00 P.M. (lights off) to 8:00-9:00 A.M. (lights on). Behavioral state was continuously monitored using videoEEG, and additional polysomnographic measurements were recorded for sleep staging. Data used for analysis were collected a minimum of $5 \mathrm{~d}$ after surgical placement of the electrodes to avoid, as much as possible, those perturbations associated with the surgery. Signals were amplified, filtered (band-pass, $0.3-70 \mathrm{~Hz}$ ), and digitized $(256 \mathrm{~Hz})$ with a Bio-Logic system (Bio-Logic, Mundelein, IL). Scoring of sleep stages was performed according to standard criteria (Rechtschaffen and Kales, 1968). $\theta$ oscillations were clearly visible during spontaneous awakenings and in REM sleep, as well as during quiet wakefulness, while subjects were sitting or lying in their bed fully alert with eyes open but without apparent motor behavior (determined by both video-EEG and electrophysiological measurements).

To identify EEG segments containing $\theta$ oscillations across brain states, we first applied discrete-Fourier transform algorithms to the continuous 
raw signal to obtain $\theta$ power values for each consecutive artifact-free EEG epoch ( $1 \mathrm{sec}$ ) across states. Second, $\theta$ power thresholds in each brain state were determined by selecting the peak of $\theta$ power per minute and averaging these values. Thus, $\theta$ power threshold was defined as the average of $\theta$ peaks within a given state. Only EEG segments exceeding that threshold were included in additional analyses. Spectral power density was estimated using the Welch's averaged modified periodogram method (1 sec; linear detrending and Hanning windowing; MATLAB; MathWorks, Natick, MA) for each EEG epoch selected previously in each wake-sleep stage and brain region. Partial coherence analysis (Jenkins and Watts, 1968) was computed for all possible combinations of hippocampal and neocortical electrodes in those $\theta$ segments selected previously by the discrete-Fourier transform algorithm in quiet wakefulness, transition from sleep to wake, and REM sleep. This analytic approach allowed us to discriminate between the portion of the total coherence that is directly caused by both the coupling of the two specific signals and the common influence from other neural sources not involved in the analysis (Bendat and Piersol, 1980).

For analysis of the $\gamma$ component in the human hippocampal EEG and its relationship to $\theta$ phase, the signals were filtered between $\theta(4-7 \mathrm{~Hz})$ and $\gamma(30-50 \mathrm{~Hz})$ bands using finite impulse response filters, which have precisely zero phase distortion and magnitude modified by the square of the magnitude response of the filter (filtfilt procedure, MATLAB; MathWorks). The $\gamma$ component was then rectified and integrated using a 10 -point moving average procedure. The relationship between variations of $\theta$ and $\gamma$ power was estimated using coherence and $\theta$-triggered averaging of the $\gamma$ signal. For the latter, the peaks in the $\theta$-filtered EEG were identified, and both $\theta$ and $\gamma$ signals were cut into segments representing one $\theta$ cycle each and resampled (resample procedure, MATLAB; MathWorks) to standardize the window lengths.

\section{Results}

Intracranial EEG recordings were acquired from nine patients with intractable epilepsy. Three of these patients had depth hippocampal electrodes. Artifact-free EEG segments containing $\theta$ episodes were automatically selected using discrete-Fourier transform algorithms (see Materials and Methods) applied to the raw signal across the wake-sleep continuum in each patient. Figure $1 B$ illustrates locations of recording sites across all nine patients. Colored symbols represent electrodes showed statedependent $\theta$ oscillations.

In all three patients with hippocampal-depth electrodes, transient bursts of hippocampal $\theta$ waves were identified during REM sleep (Fig. $2 A$, bottom trace) by using discrete-Fourier transform algorithms (see Materials and Methods). These hippocampal REM- $\theta$ bursts showed maximal spectral power at $5 \mathrm{~Hz}$ (mean \pm $\mathrm{SD}, 4.95 \pm 0.8 \mathrm{~Hz}$ ), lasted $<3 \mathrm{sec}$ (mean $\pm \mathrm{SD}, 1.66 \pm 0.7 \mathrm{sec}$ ) (Fig. 3C), and occurred with a mean density of $10.9 \pm 7.1$ (SD) per minute. There was no correlation between these $\theta$ bursts and the phasic appearance of rapid eye movements during REM sleep $(p<0.3)$. REM- $\theta$ bursts did not show temporal association with any electrophysiological sign of epileptic activity or spatial association with recording sites overlying hippocampal sclerosis. In addition, no equivalent $\theta$ activity was observed during SWS in either hippocampal or neocortical regions. Across $30 \mathrm{~min}$ of REM sleep and 30 min of SWS per person, the three patients with depth implants within the hippocampus showed $35 \theta$ bursts in SWS, which was $<4 \%$ of the 896 bursts seen in REM (patient 1 , $p<0.0002$; patient $2, p<10^{-9}$; patient $3, p<10^{-10}$ ).

Hippocampal $\theta$ oscillations were also observed during transitions from sleep to waking, but in this case, simultaneously with neocortical $\theta$ waves (Fig. $2 A$, top trace). These $\theta$ oscillations, seen in all three patients with hippocampal-depth EEG recordings, lasted significantly longer (mean $\pm \mathrm{SE}, 7.3 \pm 2.8 \mathrm{sec}$; unpaired $t$ test, $p<10^{-9}$ ) and were slightly faster (mean \pm SE, $5.4 \pm 0.8 \mathrm{sec}$ ) than $\theta$ bursts in REM sleep.

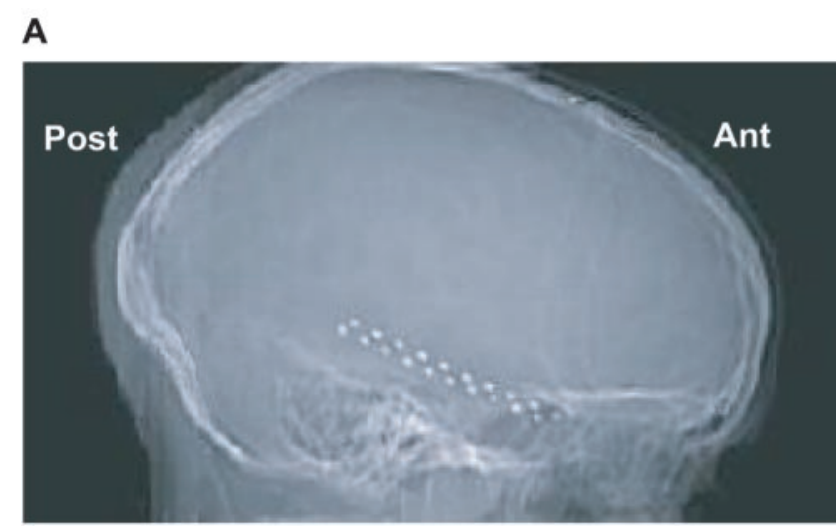

B
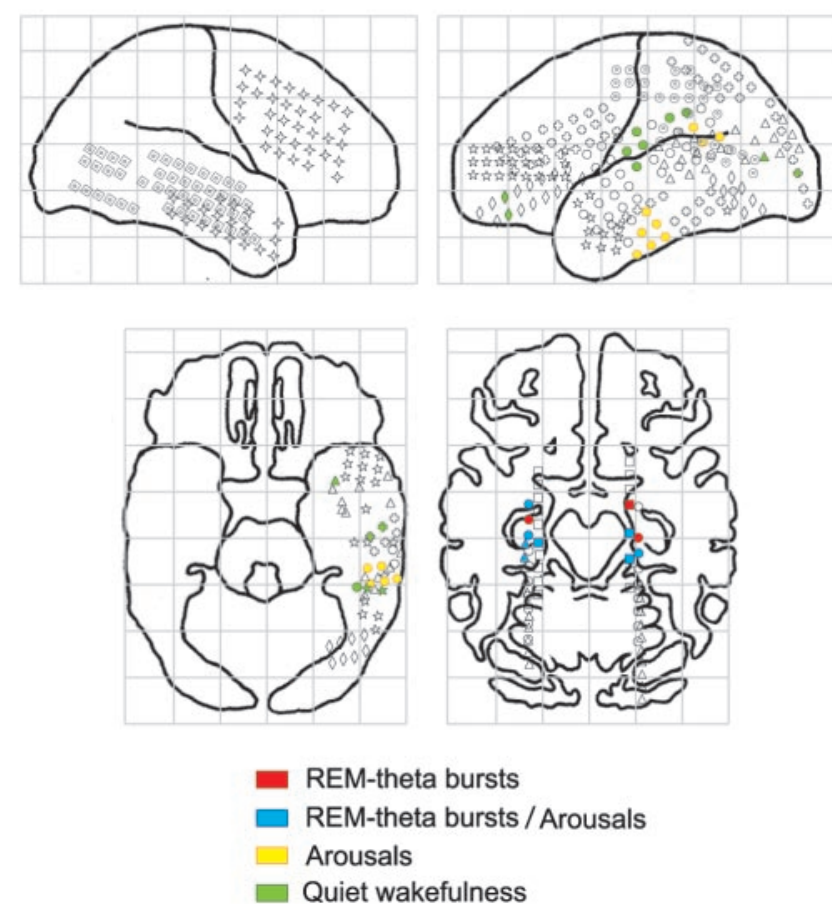

Figure 1. A, High-speed computed tomography view of the bilateral localization of multicontact depth electrodes in one of the participants. Aligned white dots correspond with the positions of the electrodes. $B$, Locations of recordings sites across all nine participants. These topographic maps show electrode locations on four views of a standard brain. Top left, Right lateral view. Top right, Left lateral view. Bottom left, Inferior view. Bottom right, Mid-axialhippocampal view. Different shapes denote locations in different participants. Color-filled shapes indicate electrodes showed state-dependent $\theta$ oscillations. Note that some similar depth electrodes (bottom right, blue) showed $\theta$ oscillations during both REM and arousals from sleep.

$\theta$ waves also appeared spontaneously in several neocortical regions during quiet wakefulness (Fig. 4). $\theta$ oscillations were clearly identified in five patients with subdural electrodes located in the frontal cortex and basal temporal lobe (Fig. $4 A, B$ ). In three additional patients who had electrodes on the surface of the parietal cortex, on lateral regions of the temporal lobe, and over occipital cortex, no spontaneous $\theta$ oscillations were observed as determined by automatic detection algorithms. Periods of neocortical $\theta$ activity during quiet wakefulness lasted significantly longer than during REM sleep in the hippocampus (mean \pm SD, $6.9 \pm 4.1 \mathrm{sec}$; unpaired $t$ test, $p<10^{-7}$ ) but were similar in duration to the neocortical $\theta$ waves associated with awakening (unpaired $t$ test; $t<1 ; \mathrm{NS}$ ). In addition, the averaged power peak 
A

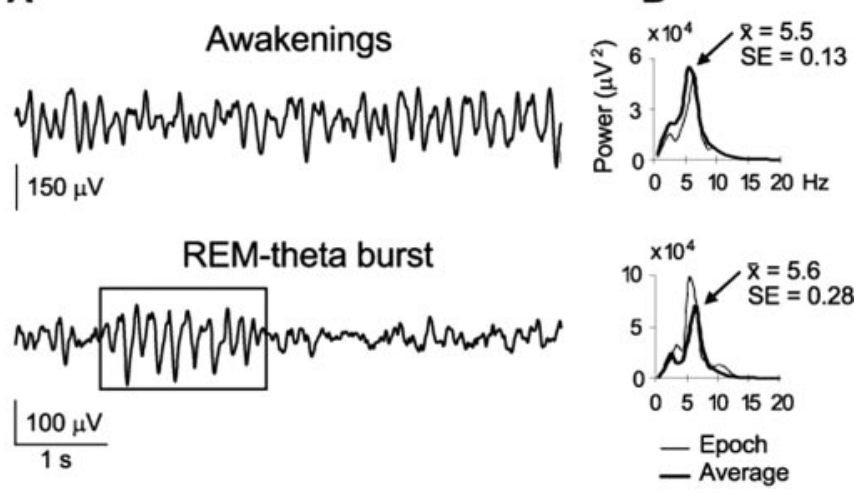

Figure 2. Human $\theta$ oscillations generated in the hippocampal formation during awakenings from natural sleep and during REM sleep in bursts. $A$, Examples of unfiltered EEG traces containing hippocampal $\theta$ oscillations associated with awakenings and REM bursts in one patient. Talairach coordinates (L-R, A-P, I-S) (in mm): (24.0, $-26.9,-7.2)$. Identical findings were observed in the remaining two patients (Talairach coordinates: 24.0, $-32.3,1.1$; and 24.0, $-32.1,-0.8)$. The same electrodes exhibited maximal $\theta$ power during awakenings and REM $\theta . B$, Spectra obtained from the EEG segments shown in $A$ (thin lines) and from averaging all $\theta$ segments (thick lines) in the same hippocampal electrode for that patient. Mean frequencies are shown for averaged segments.

A

REM

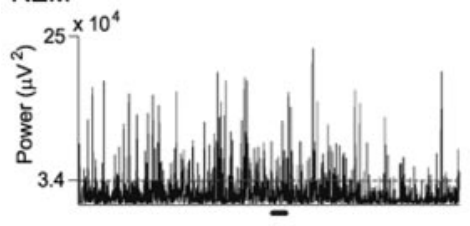

SWs

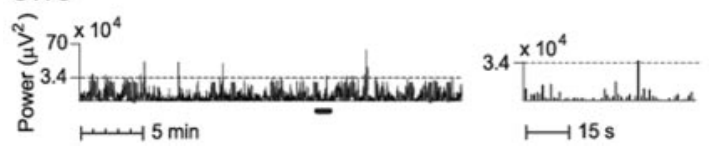

Figure 3. Comparison of $\theta$-burst activity in REM sleep and SWS. A, Representative pattern of $\theta$ power within the human hippocampus across 30 min of REM and SWS in a patient with hippocampal-depth electrodes as determined by the discrete-Fourier transform algorithms. The horizontal dashed line indicates the spectral power threshold used to quantify the number of $\theta$ bursts in each brain state. The threshold was computed using the averaged peak of $\theta$ power per minute during the entire period of each cerebral state. Talairach coordinates (L-R, A-P, I-S) (in $\mathrm{mm}$ ): $(24.0,-32.1,-0.8)$. $B$, Enlarged view of 1 min (indicated by the short, thick, black line under both plots in $A$ ) of $\theta$ power across time during REM and SWS, emphasizing the presence of short $\theta$ bursts selectively during REM sleep. C, Histogram of REM- $\theta$ burst durations computed for the same patient and recording site. Results similar to those shown in $A-C$ were obtained in the other two patients with hippocampal recordings as well.

of neocortical $\theta$ varied, depending on cortical location (Fig. 4C), although the maximum spectral power was always within the classical $\theta$ band in humans (mean $\pm \mathrm{SD}, 5.9 \pm 0.7 \mathrm{~Hz}$; frequency range, $4-7 \mathrm{~Hz}$ ). None of the three patients with depth electrodes showed $\theta$ oscillations in the hippocampus during quiet wakefulness, supporting the hypothesis that the synchronous neuronal firing associated with neocortical $\theta$ waves in humans were cortically specific and independent from $\theta$ waves generated within the hippocampus.

Because spectral energy within the $\theta$ frequency range was primarily absent from the neocortex during REM- $\theta$ bursts and from the hippocampus during quiet wakefulness (Fig. $5 B, D$ ), it seemed unlikely that these brain regions shared a single $\theta$ generator. However, the similar amounts of $\theta$ power in the neocortex
A

B

C

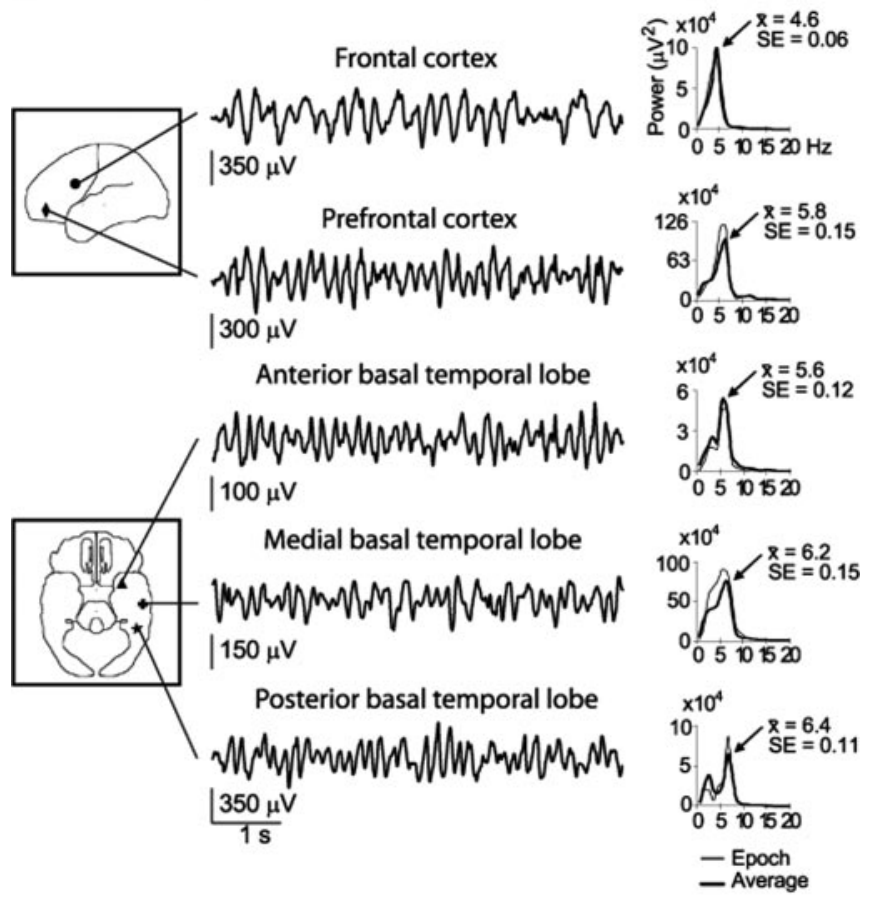

Figure 4. Human $\theta$ oscillations in different regions of the neocortex during quiet wakefulness. A, Approximate location of the electrodes that showed the maximum amplitude of $\theta$ activity in five of eight patients with neocortical recordings (each symbol denotes a different patient). Talairach coordinates (L-R, A-P, I-S) (in mm): $, 14.8,13.3,41.2 ; \bullet,-20.2,47.7$, 8.9; $\mathbf{\Delta},-29.0,0.4,-27.4 ; \mathbf{+},-60.5,-26.9,-11.4 ;$ and $\star,-44.1,-44.8,-15.7)$. $B$, Unfiltered EEG recordings from locations indicated in $A$. Note that each EEG trace belongs to a different patient and is highly representative of the pattern of electrophysiological activity observed during quiet wakefulness in each individual patient. For each patient, most of the electrodes placed within the same cortical region showed the same pattern of $\theta$ oscillations during quiet wakefulness. C, Spectral power density computed for a representative electrode in each neocortical region involved in $\theta$ generation during quiet wakefulness. Thick lines, Averaged spectra for all $\theta$ epochs; thin lines, spectra for single epochs shown in $B$. Peak frequencies are for averaged spectra.

and hippocampus during transitions into waking (Fig. $5 B, D$ ) raised the question of whether a shared generator might in fact exist. Additional analysis showed that this was not the case.

Ordinary and partial coherence analyses of the EEG waveforms from two patients with both hippocampal and neocortical electrodes yielded identical results (Fig. $5 A, C$ ). First, $\theta$ coherence between the hippocampus and neocortex was absent, regardless of the brain state (Fig. 5A,C). Second, $\theta$ oscillations within the hippocampus and neocortex (patient 1, basal regions of temporal lobe; patient 2, frontal cortex) were highly coherent during REM- $\theta$ bursts, awakenings, and quiet wakefulness, even after removing neocortical and hippocampal influences, respectively (Lopes da Silva et al., 1980; Kocsis et al., 1999). Thus, even the small amounts of $\theta$ power and local coherences observed in the hippocampus during quiet wakefulness and in the neocortex during REM were not attributable to spreading from other cerebral structures that were actively generating $\theta$ waves.

An important feature of $\theta$ states in rodents is that highfrequency oscillations in the $\gamma$ range, primarily $\sim 40 \mathrm{~Hz}$, appear in CA1 and dentate gyrus, tightly coupled with $\theta$ waves during both REM sleep and exploration. It was demonstrated in rats (Bragin et al., 1995; Csicsvari et al., 2003) and mice (Buzsaki et al., 2003) that the magnitude of $\gamma$ power maintained a phase relationship with the $\theta$ cycle. To determine whether similar phenomenon 
A

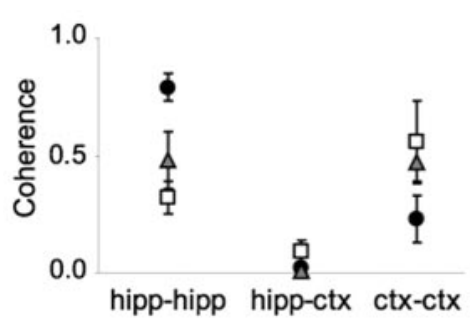

- REM-theta bursts $\Delta$ Awakenings

C

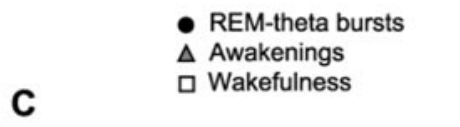

B
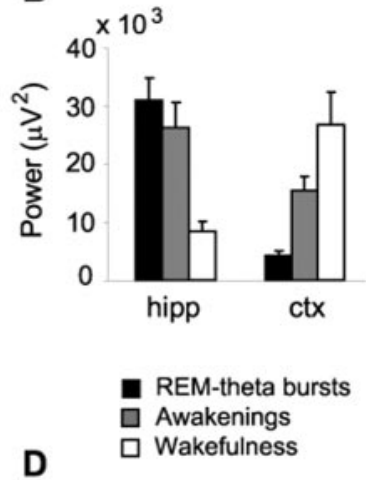

D

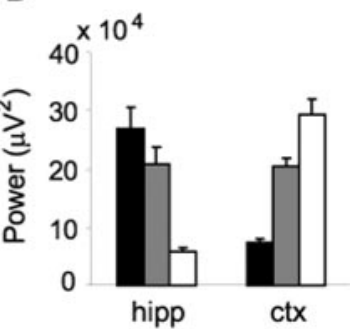

Figure 5. Evidence of different $\theta$ generator sources within the human hippocampal formation and neocortex. $A$, Averaged partial coherence results between pairs of electrodes for each wake-sleep state after removing common influences from other sources. Electrode pairs were located within the hippocampal formation (hipp-hipp; coordinates: 24.0,-26.9, -7.2 ; and $24.0,-36.2,3.1)$, the basal surface of the temporal lobe (ctx-ctx; coordinates: $-29.0,0.4$, -27.4 ; and $-43.8,-38.3,-14.6)$, or had one electrode in each structure (hipp-ctx; coordinates: $24.0,-26.9,-7.2$; and $-29.0,0.4,-27.4)$. $B$, Comparison of averaged $\theta$ power $(4-7 \mathrm{~Hz})$ within hippocampus and neocortex for each wake-sleep state. Data are from the same subject as in $A$. C, Same as $A$, but for a different patient. Corticocortical coupling in this patient was determined using a pair of electrodes located in the frontal lobe. Talairach coordinates (in mm): hipp-hipp, 24.0, -32.1, -0.8 , and 24.0, $-15.7,-12.1$; ctx-ctx, 14.8, 13.3, 41.2, and 25.0,21.9,31.2; hipp-ctx, 24.0, $-32.1,-0.8$, and 14.8, 13.3, 41.2. D, Same as B, but for the patient shown in C. Results for both partial coherence and spectral analysis were essentially unchanged when other combinations of electrodes were used in each patient.

occurs in the human hippocampus, $\gamma$ phase synchrony was investigated as a function of $\theta$ cycle for each EEG epoch containing REM- $\theta$ bursts.

We found that $30-50 \mathrm{~Hz}$ oscillations were indeed present in human hippocampal EEG during $\theta$ states. However, $\gamma$ oscillations showed no phase modulation triggered by hippocampal $\theta$ cycle (Fig. 6) in any of the three patients, which is in clear contrast with previous findings reported in rodents (Bragin et al., 1995; Buzsaki et al., 2003; Csicsvari et al., 2003).

\section{Discussion}

The findings reported here reveal that, as in other mammals, human $\theta$ oscillations ( $4-7 \mathrm{~Hz}$ ) observed within the hippocampus as well as within specific regions of the neocortex are modulated by brain state, although they also show important differences in electrophysiological properties as compared with other species. The absence of coupling between hippocampus and any neocortical region during the emergence of $\theta$ oscillations strongly suggests that these cerebral structures have distinct and independent $\theta$-wave generators, which are activated differently depending on the ongoing brain state. Furthermore, $\gamma$ amplitude did not appear to be modulated by $\theta$ cycle within the hippocampus, pointing out that generation mechanisms of both cerebral rhythms may not be shared in humans. Human $\theta$ oscillations were reported previously to be task-modulated in parietoccipital cortex and basolateral regions of the temporal lobe when patients were

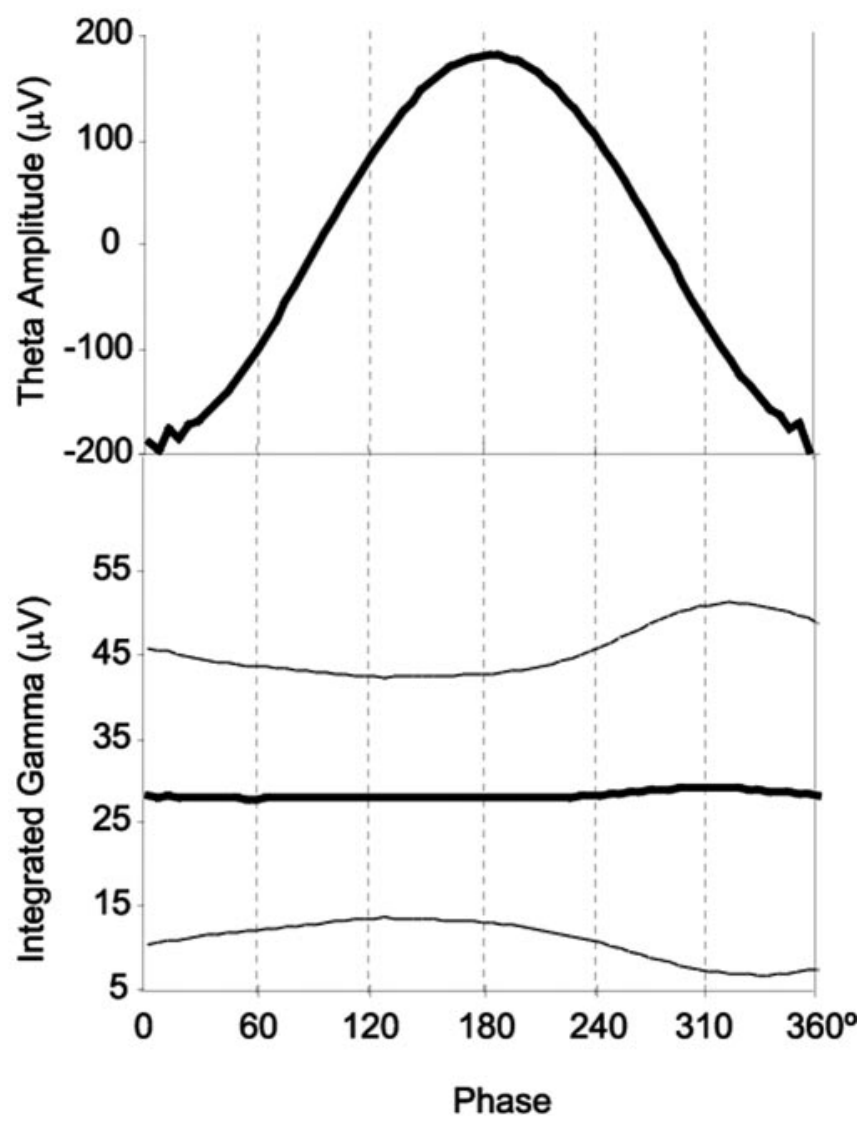

Figure 6. Absence of phase relationship between $\theta$ cycle and $\gamma$ amplitude in human hippocampus. Averaged peak of $\gamma$ amplitude (bottom, thick line) showed an inconsistent relationship with the $\theta$ cycle (top) as revealed by the high variability observed in $\gamma$ peak ( \pm SD values represented by thin lines). $\theta$ cycle (top) was obtained after averaging all $\theta$ cycles contained in REM- $\theta$ bursts recorded in three patients with hippocampal implants.

performing a virtual navigation task (Kahana et al., 1999; Caplan et al., 2001, 2003). However, $\theta$ was also observed at many sites that were not task-dependent (Kahana et al., 1999; Caplan et al., 2001). Therefore, our study supports previous findings on the existence of $\theta$ oscillations in human neocortex during wakefulness, but it also provides the first demonstration of sleepdependent $\theta$ oscillations $(4-7 \mathrm{~Hz})$ in both human neocortex and hippocampus.

Tonic oscillations in the $\delta$ range $(1.5-3 \mathrm{~Hz})$ have been reported previously to be present in temporolateral regions and hippocampus during human REM sleep. These oscillations showed a higher relative power in human REM sleep in comparison with other cerebral states (Bodizs et al., 2001). In contrast, our results revealed that human $\theta$ oscillations $(4-7 \mathrm{~Hz})$ appear in short bursts during REM sleep, and no similar electrophysiological events were observed in either SWS or wakefulness. The reason for these discrepancies is not altogether clear. Electrodes were placed in parahippocampal regions and the hippocampus using different implantation techniques in each study. Thus, our patients were implanted with multicontact depth electrodes, whereas foramen ovale electrodes were used in the study by Bodizs et al. (2001). Although both studies claim that electrodes were placed within the hippocampus, the exact location is probably different. This aspect may be especially important because $\theta$ rhythm in lower mammals has been shown to change dramatically in amplitude, depending on the hippocampal subregion or layer (Buzsaki et al., 1986; Brankack et al., 1993). Another reason 
for this inconsistency might be that phasic $\theta$ activity was never looked for in previous studies (Bodizs et al., 2001; Uchida et al., 2001), suggesting that different types of hippocampal slow oscillations might coexist in human hippocampus during REM sleep.

We used partial coherence analysis to investigate hippocampal-cortical relationships in the generation of human $\theta$ oscillations in different brain states. This analytic approach has been successfully applied to determine not only the direct correlation between EEG signals recorded from two different brain regions but also whether highly coherent oscillations within two structures are either a property of the system itself or the result of a third common input (Lopes da Silva et al., 1980; Kocsis et al., 1999). Thus, whereas the hippocampus appears to be primarily involved in the generation of REM- $\theta$ bursts, both basal regions of temporal lobe and frontal cortex participate more actively in the generation of $\theta$ oscillations during quiet wakefulness. Interestingly, cortical and hippocampal generators of $\theta$ oscillations were equally active during the transition from sleep to wakefulness. The presence of neocortical $\theta$ oscillations observed here and in other studies might be mediated by the entorhinal cortex influence. This cerebral structure has been shown to have widespread projections to different neocortical regions (Amaral and Witter, 1995), and to generate $\theta$ oscillations (Mitchell et al., 1982). Therefore, results obtained in this study support the existence of independent and state-dependent generators of human $\theta$ oscillations located in the hippocampus and the presence of statedependent $\theta$ oscillations in basal regions of the temporal lobe and frontal cortex. It is relevant to state that rodent $\theta$ rhythms, observed in a variety of species, show significant differences as compared with the electrophysiological features of human $\theta$ oscillations observed in this study, which suggests that human $\theta$ generators might have evolved and changed their functional roles as part of the brain evolutionary process.

Hippocampal $\theta$ oscillations observed during transition to wakefulness are similar to the 3-7 Hz waves reported in the hippocampus of unanesthetized rabbits (Green and Arduini, 1954), which also lasted for several seconds and were consistently associated either with spontaneous awakenings from natural sleep or with periods of waking with afferent stimulation. The similarity of these $3-7 \mathrm{~Hz}$ oscillations to the $\theta$ oscillations reported here suggests that $\theta$ activity might also serve as an arousal mechanism for the transition from sleep to wake, emerging in humans not only in the hippocampus but in cortical structures as well.

The role of hippocampal $\theta$ in animal cognition is well established. Elimination of hippocampal $\theta$ rhythm in rodents results in deficits in both acquisition (Mitchell et al., 1982) and retrieval of spatial memories (Winson, 1978). But there is only indirect evidence, coming from magnetoencephalographic studies, suggesting the possibility of human hippocampal $\theta$ oscillations during spatial navigation (De Araujo et al., 2002) and working memory (Tesche and Karhu, 2000) tasks. Systematic studies are now needed to determine whether human hippocampal $\theta$ oscillations are coupled to motor behavior and spatial cognition, because they are in rodents.

Our findings demonstrate that $\theta$ oscillations are also a prototypical feature of human REM sleep, although they appear transiently as compared with other lower mammals, and their appearance is restricted to the hippocampal formation. The phasic nature of hippocampal $\theta$ activity seen during human REM sleep is different from the tonic activity seen in rodents but similar to the short bouts of $\theta$ activity recorded from the basal dendritic side of CA1 in monkeys under urethane anesthesia (Stewart and Fox, 1991), which points again to clear differences between lower and higher mammals with respect to the brain mechanisms involved in the generation of $\theta$ oscillatory activity. It has been suggested that $\theta$ oscillations enhance the formation of long-term memories by facilitating the process of long-term potentiation (Larson and Lynch, 1986; Pavlides et al., 1988; Huerta and Lisman, 1996; Holscher et al., 1997), even when $\theta$ waves and incoming stimuli are correlated for periods $\leq 1 \mathrm{sec}$. Thus, the relatively brief REM- $\theta$ bursts recorded in the human hippocampus are compatible with a model of potentiated off-line memory consolidation during sleep. Hippocampal $\theta$ oscillations during REM sleep, together with intermittent sharp waves initiated in the hippocampus during slow-wave sleep, have been proposed as a potential neurophysiological mechanism for the preservation of experienceinduced synaptic modifications in rodents (Buzsaki, 1998). Although speculative, a possible functional role for the human REM- $\theta$ bursts reported here might be the off-line reactivation of memory traces within the hippocampus, perhaps priming the memory for replay to the neocortex during other sleep stages or in association with other electrophysiological events.

As shown in rodents in detail, state-dependent activity of the hippocampus is controlled by ascending subcortical input, including chemical modulatory drives (Vertes and Kocsis, 1997). During REM sleep, for example, both elevated acetylcholine level and serotonergic withdrawal exert $\theta$-promoting effects that last throughout the entire state of REM sleep. They activate various extra- and intra-hippocampal $\theta$ generators, which in turn produce uninterrupted rhythmic slow oscillations in the hippocampal EEG. The development of these generators through evolution is unknown. However, short periods of $\theta$ in anesthetized monkey (Stewart and Fox, 1991) and the transient nature of human REM- $\theta$ bursts shown here point to the role of intrinsic hippocampal generators, which are known to fluctuate in the rat (Kocsis et al., 1999) and produce intermittent oscillations in slice preparations in response to administration of cholinergic agonists (Konopacki et al., 1987).

Because epilepsy has been primarily proposed as a disorder of synchronization, $\theta$ oscillations reported here might be thought to be a manifestation of such pathology. Several sources of evidence lead us to believe that our findings of state-dependent $\theta$ oscillations reflect normal brain activity. (1) Electrodes overlying regions of known lesions or seizure-onset zones were excluded from analysis. (2) Sites showed $\theta$ oscillations in each cerebral state with a high degree of spatial localization (Fig. $1 B$ shows a finding opposite of what one would expect from an uncontrolled process like epilepsy). (3) Brain regions showing $\theta$ oscillations in waking and sleep were quite different, a finding difficult to explain in terms of epileptic foci. (4) Variants of oscillatory activity present in epileptic brains have been shown previously to be unrelated to the pathology. One illustrative example is sleep spindles, which have been recorded intracranially in epileptic patients and shown to be similar to normal sleep spindles but unrelated to epilepsy (Malow et al., 1999). (5) $\theta$ oscillations may actually act to inhibit seizure onset in vivo (Miller et al., 1994), and $\theta$ in vitro is insensitive to osmolality, a variable that affects patients' susceptibility to seizures (Osehobo and Andrew, 1993). (6) None of the participants showed electrophysiological signs of epileptic activity during sleep states. (7) The presence of hippocampal REM- $\theta$ bursts was observed in three of three patients with hippocampal depth implants. It is important to note that one of these (patient 5) (Table 1) did not have an epileptic focus within the hippocampus, and the remaining two (patients 1 and 4) (Table 1) did not show REM- $\theta$ bursts in electrodes placed over regions that had 
radiographically evident structural damage or that exhibited epileptiform activity.

The findings presented in this study support the hypothesis that hippocampal $\theta$ oscillations are a ubiquitous, statedependent, cerebral manifestation maintained across much of the mammalian class. It is tantalizing to consider the possibility that the appearance of independent $\theta$ generators in the human cortex might contribute to our unique cognitive capacities.

\section{References}

Amaral DG, Witter MP (1995) Hippocampal formation. In: The rat nervous system, Ed 2 (Paxinos G, ed), pp 443-493. Sidney: Academic.

Arnolds DE, Lopes da Silva FH, Aitink JW, Kamp A, Boeijinga P (1980) The spectral properties of hippocampal EEG related to behaviour in man. Electroencephalogr Clin Neurophysiol 50:324-328.

Bendat JS, Piersol AG (1980) Engineering applications of correlation and spectral analysis. New York: Wiley.

Berry SD, Thompson RF (1978) Prediction of learning rate from the hippocampal electroencephalogram. Science 200:1298-1300.

Bland BH, Oddie SD (2001) Theta band oscillation and synchrony in the hippocampal formation and associated structures: the case for its role in sensorimotor integration. Behav Brain Res 127:119-136.

Bodizs R, Kantor S, Szabo G, Szucs A, Eross L, Halasz P (2001) Rhythmic hippocampal slow oscillation characterizes REM sleep in humans. Hippocampus 11:747-753.

Bragin A, Jando G, Nadasdy Z, Hetke J, Wise K, Buzsaki G (1995) Gamma $(40-100 \mathrm{~Hz})$ oscillation in the hippocampus of the behaving rat. J Neurosci 15:47-60.

Brankack J, Stewart M, Fox SE (1993) Current source density analysis of the hippocampal theta rhythm: associated sustained potentials and candidate synaptic generators. Brain Res 615:310-327.

Buzsaki G (1998) Memory consolidation during sleep: a neurophysiological perspective. J Sleep Res 7:17-23.

Buzsaki G (2002) Theta oscillations in the hippocampus. Neuron 33:325-340.

Buzsaki G, Czopf J, Kondakor I, Kellenyi L (1986) Laminar distribution of hippocampal rhythmic slow activity (RSA) in the behaving rat: current source density analysis. Brain Res 365:125-137.

Buzsaki G, Buhl DL, Harris KD, Csicsvari J, Czeh B, Morozov A (2003) Hippocampal network patterns of activity in the mouse. Neuroscience 116:201-211.

Caplan JB, Madsen JR, Raghavachari S, Kahana MJ (2001) Distinct patterns of brain oscillations underlie two basic parameters of human maze learning. J Neurophysiol 86:368-380.

Caplan JB, Madsen JR, Schulze-Bonhage A, Aschenbrenner-Schebe R, Newman EL, Kahana MJ (2003) Human $\theta$ oscillations related to sensorimotor integration and spatial learning. J Neurosci 23:4726-4736.

Csicsvari J, Jamieson B, Wise KD, Buzsaki G (2003) Mechanisms of gamma oscillations in the hippocampus of the behaving rat. Neuron 37:311-322.

De Araujo DB, Baffa O, Wakai RT (2002) Theta oscillations and human navigation: a magnetoencephalography study. J Cogn Neurosci 14:70-78.

Freemon FR, Walter RD (1970) Electrical activity of human limbic system during sleep. Compr Psychiatry 11:544-551.

Green JD, Arduini AA (1954) Hippocampal electrical activity in arousal. J Neurophysiol 17:533-557.

Halgren E, Babb TL, Crandall PH (1978) Human hippocampal formation EEG desynchronizes during attentiveness and movement. Electroencephalogr Clin Neurophysiol 44:778-781.

Holscher C, Anwyl R, Rowan MJ (1997) Stimulation on the positive phase of hippocampal $\theta$ rhythm induces long-term potentiation that can be depotentiated by stimulation on the negative phase in area CAl in vivo. J Neurosci 17:6470-6477.

Huerta PT, Lisman JE (1996) Bidirectional synaptic plasticity induced by a single burst during cholinergic theta oscillation in CA1 in vitro. Neuron 15:1053-1063.

Jenkins GM, Watts DG (1968) Spectral analysis and its applications. Merrifield, VA: Holden-Day.

Kahana MJ, Sekuler R, Caplan JB, Kirschen M, Madsen JR (1999) Human theta oscillations exhibit task dependence during virtual maze navigation. Nature 399:781-784.
Kocsis B, Bragin A, Buzsaki G (1999) Interdependence of multiple $\theta$ generators in the hippocampus: a partial coherence analysis. J Neurosci 19:6200-6212.

Konopacki J, McIver MB, Bland BH, Roth SH (1987) Carbachol-induced EEG "theta" activity in hippocampal brain slices. Brain Res 405:196-198.

Larson J, Lynch G (1986) Induction of synaptic potentiation in hippocampus by patterned stimulation involves two events. Science 232:985-988.

Lopes da Silva FH, Vos JE, Mooibroek J, van Rotterdam A (1980) Relative contributions of intracortical and thalamo-cortical processes in the generation of alpha rhythms, revealed by partial coherence analysis. Electroencephalogr Clin Neurophysiol 50:449-456.

Malow BA, Carney PR, Kushwaha R, Bowes RJ (1999) Hippocampal sleep spindles revisited: physiologic or epileptic activity? Clin Neurophysiol 110:687-693.

Meador KJ, Thompson JL, Lorin DW, Murro AM, King DW, Gallagher BB, Lee GP, Smith JR, Flanigin HF (1991) Behavioral state-specific changes in human hippocampal theta activity. Neurology 41:869-872.

Miller JW, Turner GM, Gray BC (1994) Anticonvulsant effects of the experimental induction of hippocampal theta activity. Epilepsy Res 18:195-204.

Mitchell SJ, Rawlins JN, Steward O, Olton DS (1982) Medial septal area lesions disrupt $\theta$ rhythm and cholinergic staining in medial entorhinal cortex and produce impaired radial arm maze behavior in rats. J Neurosci 2:292-302.

O'Keefe J, Recce ML (1993) Phase relationship between hippocampal place units and the EEG theta rhythm. Hippocampus 3:317-330.

Osehobo EP, Andrew RD (1993) Osmotic effects upon the theta rhythm, a natural brain oscillation in the hippocampal slice. Exp Neurol 124:192-199.

Pavlides C, Greenstein YJ, Grudman M, Winson J (1988) Long-term potentiation in the dentate gyrus is induced preferentially on the positive phase of theta-rhythm. Brain Res 439:383-387.

Raghavachari S, Kahana MJ, Rizzuto DS, Caplan JB, Kirschen MP, Bourgeois B, Madsen JR, Lisman JE (2001) Gating of human $\theta$ oscillations by a working memory task. J Neurosci 21:3175-3183.

Rechtschaffen A, Kales A (1968) A manual of standardized terminology, techniques, and scoring system for sleep stages of human subjects. Los Angeles: UCLA Brain Information Service-Brain Research Institute.

Rizzuto DS, Madsen JR, Bromfield E, Schulze-Bonhage A, Seelig D, Aschenbrenner-Scheibe R, Kahana MJ (2003) Reset of human neocortical oscillations during a working memory task. Proc Natl Acad Sci USA 100:7931-7936.

Seager MA, Johnson LD, Chabot ES, Asaka Y, Berry SD (2002) Oscillatory brain states and learning: impact of hippocampal theta-contingent training. Proc Natl Acad Sci USA 99:1616-1620.

Skaggs WE, McNaughton BL, Wilson MA, Barnes C (1996) Theta phase precession in hippocampal neuronal populations and the compression of temporal sequences. Hippocampus 6:149-172.

Stewart M, Fox SE (1991) Hippocampal theta activity in monkeys. Brain Res 538:59-63.

Talairach J, Tournoux P (1988) Co-planar stereotaxic atlas of the human brain. Stuttgart: George Thieme Veerlag.

Tesche CD, Karhu J (2000) Theta oscillations index human hippocampal activation during a working memory task. Proc Natl Acad Sci USA 97:919-924.

Uchida S, Maehara T, Hirai N, Okubo Y, Shimizu H (2001) Cortical oscillations in human medial temporal lobe during wakefulness and all-night sleep. Brain Res 891:7-19.

Vanderwolf CH (1969) Hippocampal electrical activity and voluntary movement in the rat. Electroencephalogr Clin Neurophysiol 27:407-418

Vertes RP, Kocsis B (1997) Brainstem-diencephalo-septohippocampal systems controlling the theta rhythm of the hippocampus. Neuroscience 81:893-926.

Whishaw IQ, Vanderwolf CH (1973) Hippocampal EEG and behavior: changes in amplitude and frequency of RSA (theta rhythm) associated with spontaneous and learned movement patterns in rats and cats. Behav Biol 8:461-484.

Winson J (1972) Interspecies differences in the occurrence of theta. Behav Biol 7:479-487.

Winson J (1978) Loss of hippocampal theta rhythm results in spatial memory deficit in the rat. Science 201:160-163. 